\title{
Chain based Routing Protocol for Wireless Sensor Network
}

\author{
Bhavna Patel \\ Student, Department of Electronics and \\ Communication Engineering, Uka Tarsadia \\ University, Gujarat.
}

\author{
Jayesh Munjani \\ Assistant Professor,Department of Electronics and \\ Communication Engineering, Uka Tarsadia \\ University, Gujarat.
}

\begin{abstract}
Wsn Formed by hundreds or thousands of heterogeneous sensor node (motes) devices spread over large field that share or exchange information with each other and move data along from one to another nodes. But in reality, these wireless networks are composed of resource constrained tiny sensor nodes. The resource constrained nature of WSN creates different kinds of challenges in its operations and design that degrading its performance. In wireless sensor network, one of the important issues is inherent limited battery power of the sensor node within network. There are different routing protocols that have been proposed for this issue. Energy consumption and network lifetime are the major issues in Wireless Sensor Networks (WSN). There are two competing objectives in the design of WSN. First objective is reduced energy consumption and second objective is increase the lifetime of network. Because of this reason PEGASIS protocol and multi-chain PEGASIS Protocol can be selected for better performance in terms of energy efficiency and network life time.
\end{abstract}

\section{Keywords}

Wireless sensor networks, Routing protocols, PEGASIS, Multi-chain PEGASIS, Energy efficiency, Network lifetime.

\section{INTRODUCTION}

Wireless sensor networks (WSN) consisting of small devices, which collect information by cooperating with each other. These small sensing devices are called nodes and consist of CPU (for data processing), memory (for data storage), battery (for energy) and transceiver (for receiving and sending signals or data from one node to another). The size of each sensor node varies with applications. For example, in some military or surveillance applications it might be microscopically small. Its cost depends on its parameters like memory size, processing speed and battery.

Today, wireless sensor networks are widely used in the commercial and industrial areas such as environmental monitoring, habitat monitoring, healthcare, process monitoring and surveillance. For example, in a military area, we can use wireless sensor networks to monitor an activity. If an event is triggered, these sensor nodes sense it and send the information to the base station (called sink) by communicating with other nodes [7].

In sensor network there is a BS (base station) which is located far away from the sensor field. Sensor nodes send the sensed data to the BS. For sending the sensed data to BS directly a lot of energy is consumed. So it is desirable to develop some routing protocols .Energy conservation and maximization of network lifetime are the key challenges in the design and implementation of WSNs [1][2].
The general architecture and the major components of a wireless sensor device (node) are in Figure. A wireless sensor device is generally composed of four basic components: a sensing unit, a processing unit, a transceiver unit and a power unit.

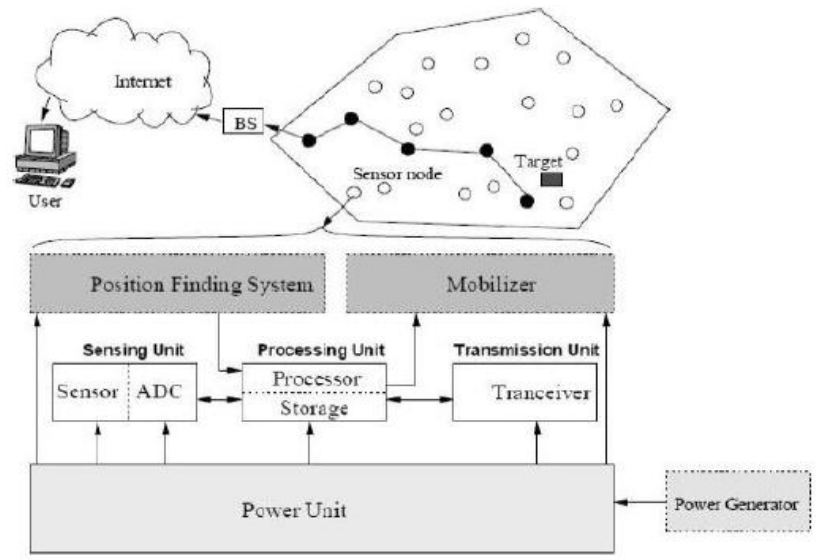

Fig 1: The general architecture of sensor networks [3]

The sensor unit consists of sensor and ADC that is Analog to Digital Converter. The sensor unit is responsible for collecting information as the ADC requests, and returning the analog data it sensed. ADC is a translator that tells the CPU what the sensor unit has sensed, and also informs the sensor unit what to do. Communication unit is tasked to receive command or query from and transmit the data from CPU to the outside world. CPU is the most complex unit. It interprets the command or query to ADC, monitors and controls power if necessary, processes received data, computes the next hop to the sink, etc. Power unit supplies power to sensor unit, processing unit and communication unit. Each node may also consist of the two optional components namely Location finding system and Mobilizer. If the user requires the knowledge of location with high accuracy then the node should uses Location finding system and Mobilizer is used to enable node movement.

\section{APPLICATION OF WSNS}

Some of the applications of sensor networks are:

1) Military: Military situation awareness [6], Sensing intruders on basis. Battle field surveillances [5].

2) Emergency situation: Disaster management, Fire/water detector [4], Hazardous chemical level and fires [3].

3) Physical world: Habitual monitoring, Observation of biological and artificial systems, Environmental monitoring of water and soil. 
4) Medical and health: Sensors for blood flow, respiratory rate, ECG (electrocardiogram), pulse oxymeter, blood pressure and oxygen measurement, Monitoring people's location and health condition [5].

5) Industrial: Factory process control and industrial automation [6], Monitoring and control of industrial equipment [3], Manufacturing monitoring [5].

6) Home network: Home appliances, location awareness (Bluetooth), Person locator.

7) Automotive: Tire pressure monitoring [3], Active mobility, and coordinated vehicle tracking [6]

8) Ocean: Monitoring fish [5].

\section{RELATED WORK}

In this paper, we propose a new method for reducing the energy consumption. Among the hierarchical protocols, LowEnergy Adaptive Clustering Hierarchy (LEACH) and Power Efficient Gathering in Sensor Information Systems (PEGASIS) are the most famous. We simulate our new method using MATLAB R2011b software and we will see the improvement in proposed protocol.

In the recent years many energy efficient chain based routing protocol has been proposed some of the major protocols are given below.

\subsection{PEGASIS Protocol}

PEGASIS protocol which was introduced by Lindsey Stephanie [8], is a power-efficient gathering in sensor information systems. This protocol is a chain based routing protocol. In PEGASIS Protocol, all nodes communicate with their closest neighbors and continue their communication until the aggregated data reached the BS. Thus this protocol improves the network lifetime, since it reduces the power consumption required per round. The difference from leach is to use multi-hop routing by forming chains and selecting only one node transmit to the Base station instead of using multiple nodes.

The key features of PEGASIS are [3]

- The BS is fixed at a far distance from the sensor nodes.

- The sensor nodes (motes) having homogeneous capability and energy constrained with uniform energy.

- No mobility of sensor nodes.

\subsubsection{PEGASIS Algorithm Detail}

1. Chain Formation: To construct the chain in PEGASIS protocol starts from the furthest node from the BS and uses Greedy algorithm to form a chain. The main idea here is that each sensor node communicates only with its closest two neighbors in order to minimize the power consumption [9].

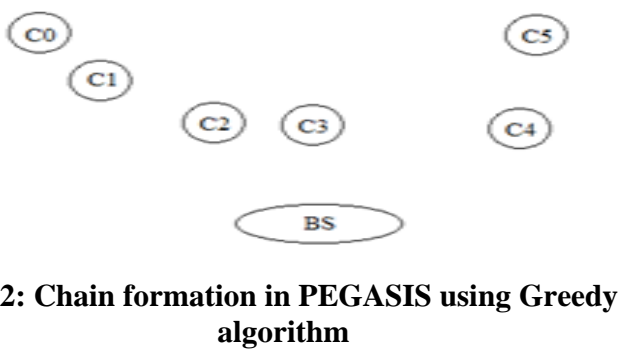

In Fig. 2node $\mathrm{C} 0$ lies the furthest from the base station so the chain construction starts from $\mathrm{C} 0$ which is connected to node $\mathrm{C} 1, \mathrm{C} 1$ is connected to node $\mathrm{C} 2$, and so on till C5.

2. Leader Selection: At the beginning of each round, a chain leader is selected randomly. This way of selection is easy and fast since no extra computation is performed. Moreover, the random selection has the benefit that when nodes is to die at random locations to providing robust network. After the leader has been selected it passes a token message to initiate a data gathering process. Passing a token also consumes energy however, the cost of passing a token is very small since the size of the token message is very small [9].

3. Data Transmission: Per round, Gathering the data in each round, each node Gathering or receives data from near neighbor, fuses its own data with it, and transmits it to the other neighbor on the chain until the whole chain data reaches the chain leader. Finally, the chain leader sends this data to the BS. Fig.3 shows a simple example for data transmission in PEGASIS protocol. First the chosen leader C3 sends a token to all the nodes in the chain. Immediately after the chain nodes receive the token both nodes $\mathrm{C} 0$ and $\mathrm{C} 5$ (the two ends of the chain) start sending their data to $\mathrm{C} 1$ and $\mathrm{C} 4$ respectively and fuse their data with the received data to $\mathrm{C} 2$ and $\mathrm{C} 3$ respectively. Then in turn, $\mathrm{C} 2$ fuses its data with $\mathrm{C} 1$ 's data and sends it to $\mathrm{C} 3$. After that Leader node $\mathrm{C} 3$ fuses its data with the data received from both $\mathrm{C} 2$ and $\mathrm{C} 4$ and sends it to the BS [9].

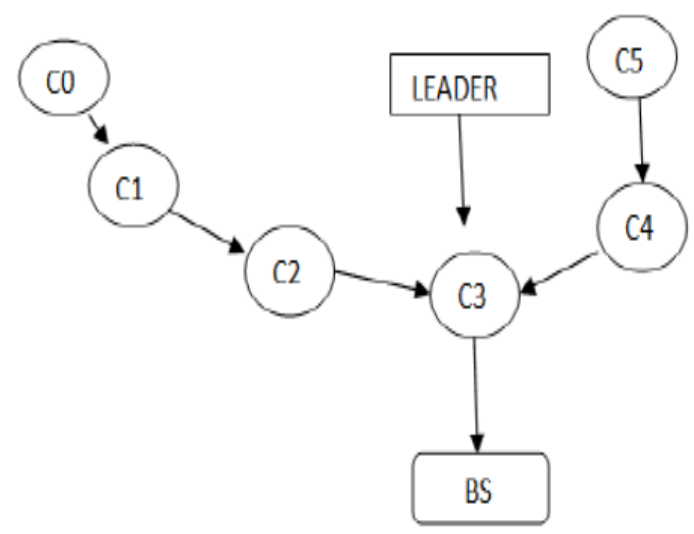

Fig 3: Data transmission

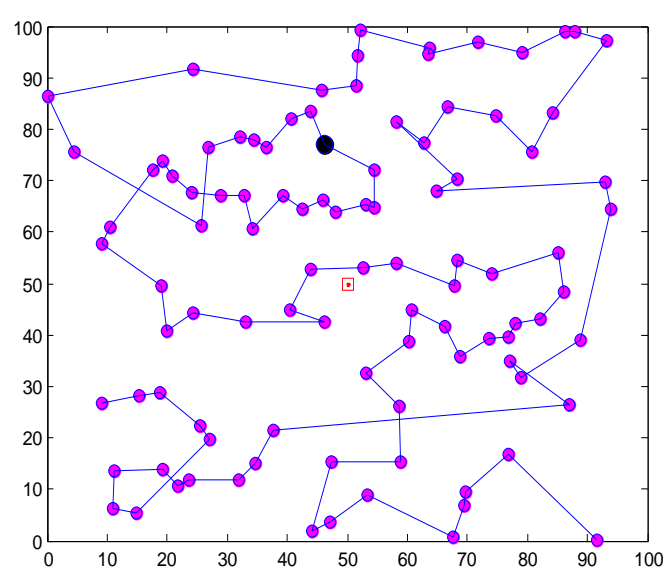

Fig 4: Chain Construction in PEGASIS protocol 


\section{MULTI-CHAIN PEGASIS (PROPOSED) PROTOCOL}

The MULTI-CHAIN PEGASIS gives better result than normal PEGASIS. Multi-chain PEGASIS approach divides the single chain into multiple parts. In this method nodes are divided in several regions and different chains are made for each region and each chain leader transmits data to Basestation. This approach gives better performance than PEGASIS.

\subsection{Chain Formation in proposed protocol}

In multi chain PEGASIS the chain formation is same as the PEGASIS except in multi-chain PEGASIS nodes are distributed in four region and each region contain a separate chain i.e 100 nodes area is divided in each $25-25$ nodes. Chain construction of proposed algorithm is shown in Fig.5.

1. Sink find the far node by comparing the distances of all nodes from itself in first region.

2. The chain construction is start from end node which is far from the sink.

3. The end node finds the most near neighbor and makes the chain between end node and nearer node.

4. Each node finds the distance between itself and the nearest node which is not repeated in connected chain and connects it with the same method which mention above.

5. The same procedure of chain formation is applied in all four regions.

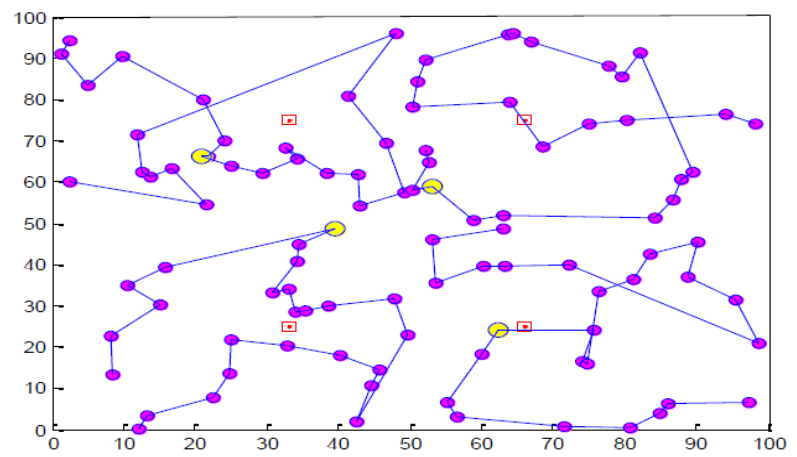

Fig 5: Chain construction and Leader selection in Proposed Protocol

\section{NETWORK MODEL}

We employ radio model to calculate energy consumption in data transmission by sensors.

To transmit m-bit packet to a node with d meters distance from the source.

Then the energy consumed will be,

$$
\mathrm{E}_{\mathrm{TX}}= \begin{cases}\mathrm{m} * \mathrm{E}_{\text {ele }}+\mathrm{E}_{\mathrm{fs}} * \mathrm{~d}^{2} & \text { if } \mathrm{d}<\mathrm{d}_{0} \\ \mathrm{~m} * \mathrm{E}_{\text {ele }}+\mathrm{E}_{\mathrm{mp}} * \mathrm{~d}^{4} & \text { if } \mathrm{d}>\mathrm{d}_{0}\end{cases}
$$

Where $d_{0}=\sqrt{\frac{E_{\mathrm{fs}}}{E_{\mathrm{mp}}}}$

To receive m-bit packet, the dissipated energy is given by,

$$
\mathrm{E}_{\mathrm{RX}}=\mathrm{m} * \mathrm{E}_{\text {ele }}
$$

Where $E_{\text {ele }}$ is energy required for electronic circuitry, $E_{\mathrm{fs}}$ is free space energy model, $\mathrm{E}_{\mathrm{mp}}$ is the multipath energy model, $\mathrm{d}_{0}$ the threshold distance, $\mathrm{E}_{\mathrm{TX}}$ is total transmitter energy, $E_{R X}$ is receiver energy.

\section{RESULT ANALYSIS \\ 6.1 Simulation environment}

Simulation has been done in MATLAB R2011b , installed on Intel(R) Core ${ }^{\mathrm{TM}}$ i3-350M CPU @ 2.4Ghz having 4GB RAM. Here for simulation $100 \times 100 \mathrm{~m}$ area is selected and 100 numbers of nodes are deployed in the network. Results have been calculated for PEGASIS and Multi-chain PEGASIS protocol with different topologies and energy. Results also show the no of dead node per round, number of alive node per round and energy consumption per round for both PEGASIS and Multi-chain PEGASIS.

\subsection{Simulation Parameters}

\begin{tabular}{|c|c|}
\hline Simulation Parameter & Value \\
\hline Network area & $100 \times 100$ \\
\hline No of nodes & 100 \\
\hline Base Station Location & PEGASIS: $(50,50)$ \\
& Proposed : BS1 $(33,25)$, \\
& BS2 $(33,75)$ BS3,(66,25), \\
& BS4 (66,75) \\
\hline Initial Energy of Nodes & $50^{*} 10^{\wedge}(-9)$ \\
\hline$E_{T X}$ & $50^{*} 10^{\wedge}(-9)$ \\
\hline$E_{R X}$ & $1.3^{*}\left(10^{\wedge}(-15)\right)$ \\
\hline$E_{a m p}$ & $10^{*}\left(10^{\wedge}(-12)\right)$ \\
\hline$E_{f s}$ & $5^{*}\left(10^{\wedge}(-9)\right)$ \\
\hline$E_{d a}$ &
\end{tabular}

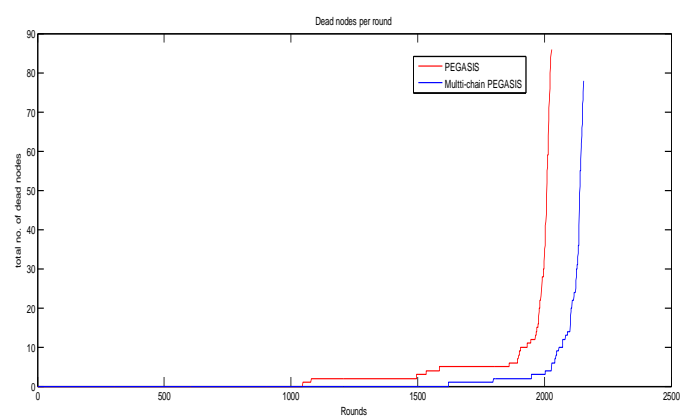

Fig 6: Rounds Vs total no of dead nodes

Fig. 6 is the implementation of PEGASIS and multi-chain PEGASIS. The graph shows the comparison of number of dead nodes to the number of rounds. After same round, the number of dead nodes starts increasing linearly.10\%node is died after 1907 round in PEGASIS and in Multi-chain PEGASIS $10 \%$ node died after 2060 rounds. In PEGASIS $50 \%$ node die after 2009 rounds and in Multi-chain PEGASIS $50 \%$ node die after 2123 round. Last node die after 2028 rounds in PEGASIS and 2147 rounds in Multi-chain PEGASIS. 


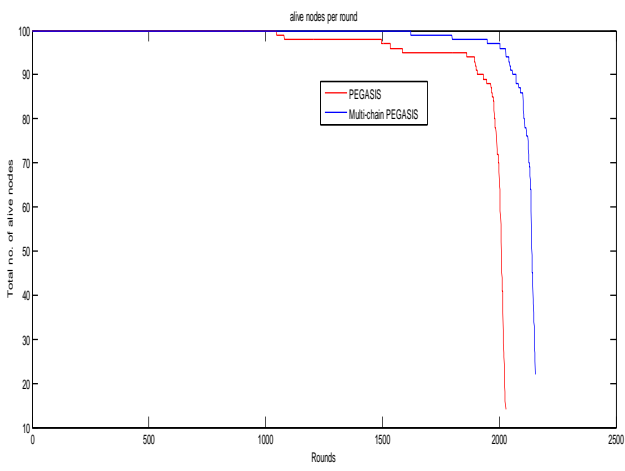

Fig 7:Rounds Vs total no of alive nodes

Fig. 7 is the implementation of PEGASIS and multi-chain PEGASIS. The graph shows the comparison of number of alive nodes to the number of rounds. After same round, the number of alive nodes starts decreasing linearly.

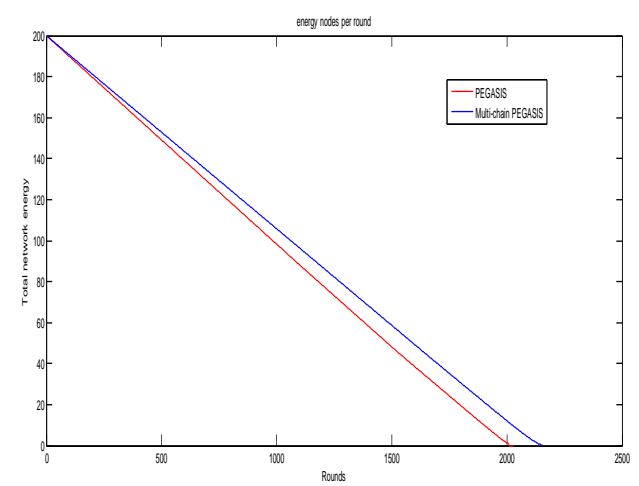

Fig 8: Rounds Vs total network energy

From Fig $8 \mathrm{We}$ observe that initially sum of energy of all nodes are 200J, as rounds are increases energy is consumed. Energy utilization is better in Multi-chain PEGASIS and hence network lifetime is increase using Multi-chain PEGASIS .Multi-chain PEGASIS is better than PEGASIS.

Table 6.1 Comparison of PEGASIS and multi-chain PEGASIS for energy $=\mathbf{2} \mathrm{J}$

\begin{tabular}{|c|c|c|}
\hline \multirow{2}{*}{$\begin{array}{l}\text { Number of dead } \\
\text { Nodes }\end{array}$} & \multicolumn{2}{|c|}{ Number of rounds } \\
\cline { 2 - 3 } & $\begin{array}{l}\text { PEGASIS } \\
\text { Protocol }\end{array}$ & $\begin{array}{l}\text { MULI- } \\
\text { CHAIN } \\
\text { PEGASIS } \\
\text { Protocol }\end{array}$ \\
\hline 10 & 1907 & 2060 \\
\hline 50 & 2009 & 2123 \\
\hline 100 & 2028 & 2147 \\
\hline
\end{tabular}

\section{CONCLUSION}

Multi-chain PEGASIS routing approach increase lifetime of sensor networks in terms of total number of dead nodes per numbers of rounds, total number of alive nodes per numbers of rounds and Total network energy per number of rounds. In this proposed scheme the longer chains have been replaced with the smaller multi chains which reduce the load on the chain leader .Multi-chain PEGASIS achieves balance of energy dissipation among the chains unlike of a single chain in PEGASIS. Multi-chain PEGASIS proved performance by using simulation on MATLAB R2011b. This proposed approach gives better performance than existing PEGASIS.

\section{REFERENCES}

[1] Navdeep Kaur, Deepika Sharma and Prabhdeep Singh "Classification of Hierarchical Routing Protocols in Wireless Sensor Network: A Survey" International Journal of P2P Network Trends and Technology, Volume 3, Issue 1- 2013.

[2] Sarjoun S. Doumit, Dharma P. Agrawal "SelfOrganizing and Energy-Efficient Network of Sensors", IEEE, pp. 1-6 (2002).

[3] 3.Samira Kalantary, Sara Taghipour"A survey on architectures, protocols, applications, and management in wireless sensor networks. "Journal of Advanced Computer Science \& Technology 3.1 (2014): 1-11.

[4] José A. Gutierrez, Marco Naeve, Ed Callaway, Monique Bourgeois, Vinay Mitter, Bob Heile, "IEEE 802.15.4: A Developing Standard for Low-Power Low-Cost Wireless Personal Area Networks" IEEENetwork, pp. 12-19 (September/October 2001).

[5] Elaine Shi, Adrian Perrig "Designing Secure Sensor Networks" IEEE Wireless Communications, pp. 38-43, December 2004.

[6] Chien-Chung Shen, Chavalit Srisathapornphat, Chaiporn Jaikaeo "Sensor Information Networking Architecture andApplications" IEEE Personal Communications, pp. 52-59, August 2001.

[7] lan F.akyildiz and mehmet can vuran "wireless sensor networks" wiley publication, 2010 .

[8] Lindsey Stephanie, and Cauligi S. Raghavendra. "PEGASIS: Power-efficient gathering in sensor information systems." Aerospace conference proceedings, Vol. 3. IEEE, 2002.

[9] Samia A. Ali and Shreen K. Refaay "Chain-Chain Based Routing Protocol", IJCSI International Journal of Computer Science Issues, Vol. 8, Issue 3, No. 2, May 2011. 